\title{
Enhancing Scatterplot Matrices for Data with Ordering or Spatial Attributes*
}

\author{
Qingguang Cui, Matthew O. Ward and Elke A. Rundensteiner \\ Computer Science Department \\ Worcester Polytechnic Institute, Worcester, MA 01609
}

\begin{abstract}
The scatterplot matrix is one of the most common methods used to project multivariate data onto two dimensions for display. While each off-diagonal plot maps a pair of non-identical dimensions, there is no prescribed mapping for the diagonal plots. In this paper, histograms, 1D plots and 2D plots are drawn in the diagonal plots of the scatterplots matrix. In 1D plots, the data are assumed to have order, and they are projected in this order. In $2 \mathrm{D}$ plots, the data are assumed to have spatial information, and they are projected onto locations based on these spatial attributes using color to represent the dimension value. The plots and the scatterplots are linked together by brushing. Brushing on these alternate visualizations will affect the selected data in the regular scatterplots, and vice versa. Users can also navigate to other visualizations, such as parallel coordinates and glyphs, which are also linked with the scatterplot matrix by brushing. Ordering and spatial attributes can also be used as methods of indexing and organizing data. Users can select an ordering span or a spatial region by interacting with 1D plots or with 2D plots, and then observe the characteristics of the selected data subset. 1D plots and 2D plots provide the ability to explore the ordering and spatial attributes, while other views are for viewing the abstract data. In a sense, we are linking what are traditionally seen as scientific visualization methods with methods from the information visualization and statistical graphics fields. We validate the usefulness of this integration by providing two case studies, time series data analysis and spatial data analysis.
\end{abstract}

Keywords: Multivariate Data Visualization, Time-series Data, Geospatial Visualization, Linked Brushing

\section{INTRODUCTION}

A scatterplot is a visual representation of data that illustrates the relation or association between two or three variables. The positions of data points represent the corresponding dimension values. Two or three dimensions are shown directly, and additional dimensions can be mapped to the color, size or shape of the plotting symbol. For visualizing multivariate data, the scatterplot matrix ${ }^{1}$ is an efficient and common tool. Given a dataset with $\mathrm{N}$ dimensions, the matrix consists of $\mathrm{N}^{2}$ scatterplots arranged in $\mathrm{N}$ rows and $\mathrm{N}$ columns. The plot in row i and column $\mathrm{j}$ uses dimension $\mathrm{i}$ and $\mathrm{j}$ to create a scatterplot. Each scatterplot reveals relationships between the two dimensions.

Since the diagonal scatterplots use the same dimension for the $\mathrm{X}$-axis and $\mathrm{Y}$-axis, the data points form a straight line of dots in these scatterplots, as shown in figure 1a. Although the straight line can show the distribution of data points in one dimension, its usefulness is very limited. Screen space is one of the most precious resources in visualization. Many layout algorithms and viewing mechanisms have been proposed to show more data or relations on the screen.

Some researchers display the labels for dimensions in the diagonal plots, while others draw histograms of individual dimensions. ${ }^{2}$ In this paper, we present a number of $0 \mathrm{D}, 1 \mathrm{D}$ and $2 \mathrm{D}$ visualizations for these diagonal plots and illustrate the usefulness of linking these views with scatterplots.

A histogram, one kind of $0 \mathrm{D}$ visualization, illustrates the distribution of a single dimension data. It conveys the data distribution much more clearly and precisely than a plot of a data dimension against itself. In 1D plots,

* This work is supported under NSF grant IIS-0119276.

Further author information: (Send correspondence to M.O. Ward)

E-mail: matt@cs.wpi.edu; Project Homepage: http://davis.wpi.edu/ $x m d v$ 


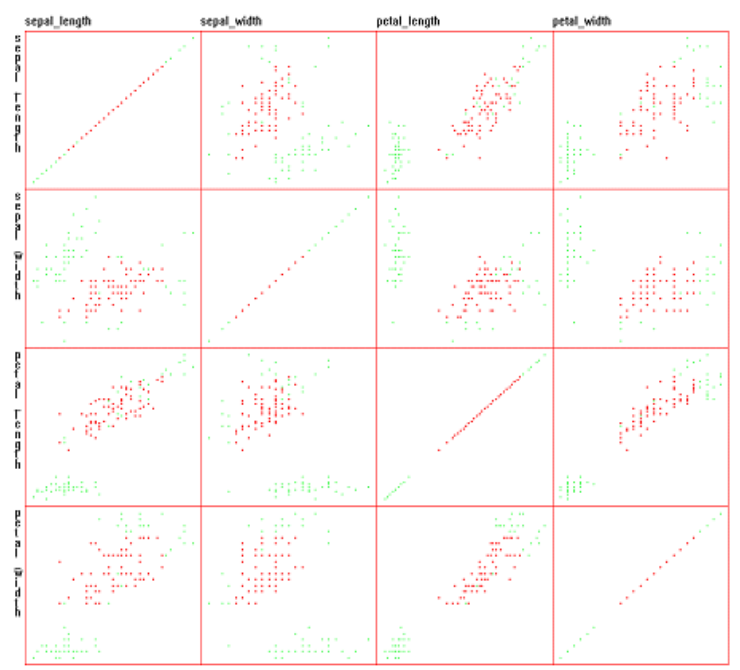

(a)

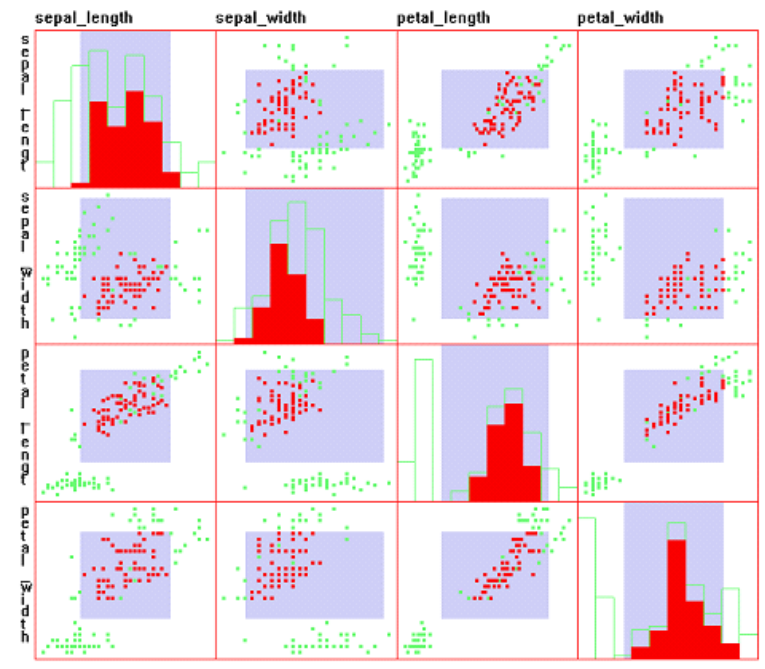

(b)

Figure 1. (a) Original Scatterplot Matrix. The data points in the diagonal plots form a straight line. (b) Scatterplot Matrix with Histograms. The diagonal plots show the histogram of each dimension. The blue boxes show the brush area; brushed data and their histograms are drawn in red.

the data are assumed to have order, which can be temporal order, one dimensional spatial order or other kinds of order (e.g. query-based ordering). The data are projected onto the screen by this order, which can convey orderbased patterns, trends and anomalies. In 2D plots, the data are assumed to have two spatial attributes, which means each data point corresponds to a particular location in $2 \mathrm{D}$ space. The dimension values are projected using a color scale.

In $2 \mathrm{D}$ plots, the spatial attributes can be provided in two ways, implicit location and explicit location. If the data are collected from a uniform grid of a two dimensional space, and the position of the data point is implicitly related to the sequence of the data records, this kind of 2D plot is called a Raster Plot. If the dataset has two dimensions that explicitly specify the location of data points, this kind of 2D plot is called a Data Driven Plot.

The implementations presented in this paper are integrated into XmdvTool, ${ }^{3}$ developed in the Computer Science Department of WPI, which is available in the public domain. It combines several of the most common methods for visualizing multivariate data, including parallel coordinates, ${ }^{4}$ scatterplot matrices, glyphs, ${ }^{5}$ dimensional stacking ${ }^{6}$ and pixel-oriented methods. ${ }^{7}$ The high dimensional brushing feature ${ }^{8}$ was added to XmdvTool to support the exploration within and between the visualizations. The structure based brushing feature ${ }^{9}$ was added to help users navigate cluster hierarchies when exploring very large datasets.

Brushing ${ }^{10}$ is a mechanism by which user can interactively select a data subset. Brushing can help users separate the interesting data from the uninteresting. For multiple views, the linking makes the brushing technique more powerful. Brushing in one view will affect the selection in other views, which links together the data points that represent the same records but are scattered in different views.

Traditional brushing operates on data values. Fua et al. ${ }^{9}$ extended the brushing operation to hierarchical structures. This paper extends the brushing operation to the ordering and spatial attributes of data. The temporal attribute is one kind of ordering attribute. The ordering and spatial attributes can be treated as other kinds of structure on which brushing is supported. Order-based brushing is implemented on the 1D plots, and spatial brushing is implemented on the 2D plots. Data brushing is also implemented in the histograms. Brushing on histograms, 1D plots and 2D plots is integrated with the brushing on other views. 


\section{RELATED WORK}

In recent years, many research efforts have focused on the integration of scientific and information visualization. Information visualization typically involves abstract data without spatial information ${ }^{11},{ }^{12}$ e.g., financial data and demographic data, which is usually found in non-scientific domains, while scientific visualization typically deals with physical data with spatial information, e.g., medical image data and geographic data, which is usually found in scientific domains. However, some datasets are not easily classified, e.g., bioinformatics data and GIS data with multiple dimensions.

Kosara et al. ${ }^{13}$ linked scientific and information visualization with interactive 3D scatterplots. The 3D scatterplots showed the three dimensional information and the abstract information at the same time, and brushing was used to highlight interesting subsets.

The WEAVE system ${ }^{14}$ linked 3D object rendering with histograms, scatterplots and parallel coordinates. The 3D object rendering show the physical characteristics of the objects, while the histogram, scatterplot and parallel coordinates illustrate the abstract patterns of the objects. They are linked by brushing. Our system differs from WEAVE in that our system assumes the dataset has ordering or spatial attributes, and then it employs $1 \mathrm{D}$ and 2D within the scatterplot matrix to show these attributes. We also allow users to control the ordering and spatial mappings to give users a wider range of views of their data. At present we do not deal with three dimensional attributes.

VizCraft ${ }^{15}$ integrated the visualization of an aircraft with a parameter set that describes the aircraft design. The visualization of the aircraft was an image of the aircraft, whose data were viewed in parallel coordinates. Our system employs 2D to show the spatial attributes, which is integrated with the scatterplot matrix and many other visualizations.

Many techniques based on hierarchical structures have been proposed to organize datasets. Cone Trees ${ }^{16}$ present hierarchical information in 3D in order to make full use of the available screen space. They also provide operations for pruning and growing the tree, reconstructing the tree dynamically, and searching the tree. Multitrees $^{17}$ are a class of visualization methods for directed acyclic graphs that can present information with multiple subtrees. These subtrees imitate models of the natural world, where many objects belong to multiple hierarchies.

\section{INTEGRATION OF SCATTERPLOTS WITH HISTOGRAMS, 1D PLOTS AND 2D PLOTS}

\subsection{Histogram}

A histogram is an aggregation method to illustrate the data distribution of a single dimension. From its minimum value to its maximum value, the data are partitioned into several sub-ranges. Each sub-range corresponds to a bin. The height of a histogram bin is decided by the number of data points whose value falls in the range associated with that bin. The number of bins is the bin count, and it influences the usability and effectiveness of a histogram. The default bin count is calculated using the formula:

$$
W=3.49 S \times N^{-\frac{1}{3}}
$$

where $\mathrm{S}$ is the standard deviation and $\mathrm{N}$ is the number of data points. It has been illustrated ${ }^{18}$ that this generally results in an effective bin count. Usually the maximum bin count for continuous data is the number that makes the bin width one pixel. The bin count can be customized from the control panel to reveal more information about the data.

On every diagonal plot of the scatterplot matrix, a dimension is used to generate a histogram. An example is shown in figure 1b. The green outline shows the regular histogram for the entire dataset corresponding to that dimension. The brushed bins (usually red) show the histograms of the data that are brushed in the scatterplots. Since each bin in the regular histogram contains at least as many data elements as the corresponding one in the brushed histogram, these two histograms can be drawn on top of each other. 


\subsection{D Plot}

Many multivariate data sets have order: financial data change over time, demographic data change by the year, and geophysical data change by the depth of stratum. A 1D Plot is a very common method to show trends and patterns in one dimensional data. The $\mathrm{x}$-axis is for the order, and the $\mathrm{y}$-axis is for the data values. The data value is drawn as a point or other graphical marker, which results in a point graph. If the consecutive points are connected together with lines, it becomes a line graph. It can also be drawn as a column graph, on which the height of each vertical bar represents a dimension value. Figure 2a shows an example of line graphs.

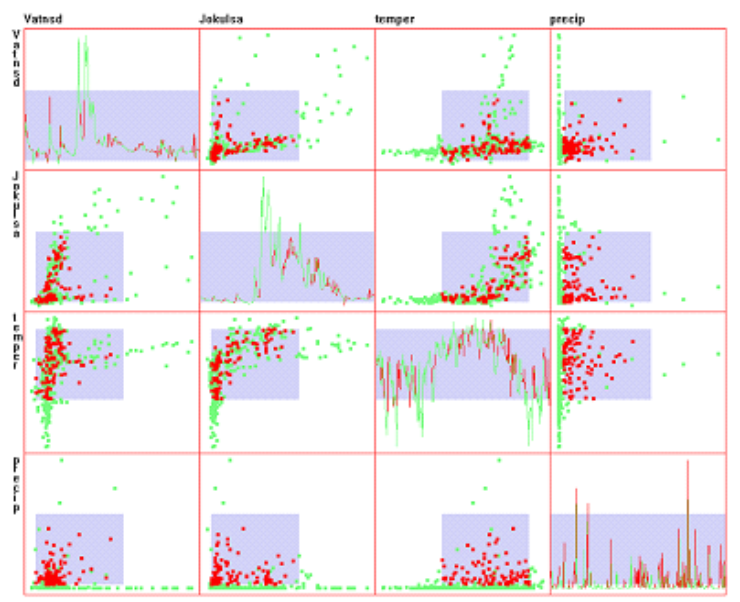

(a)

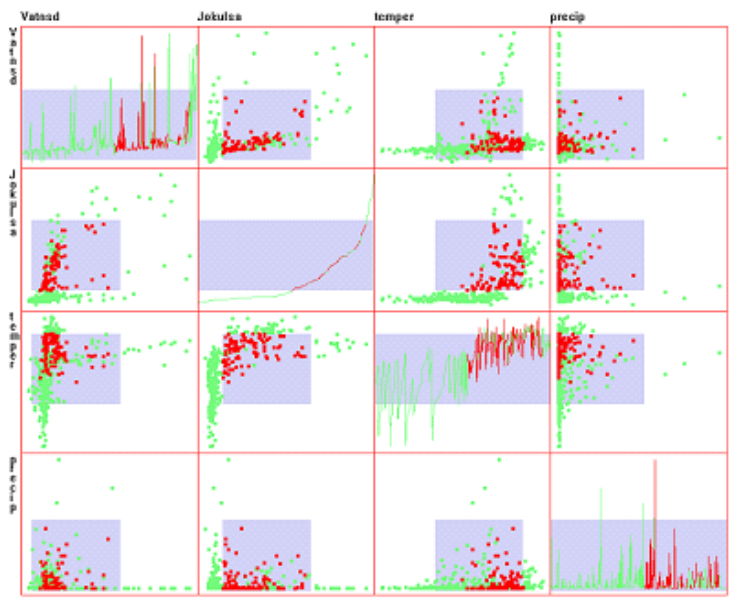

(b)

Figure 2. Scatterplot Matrix with 1D Plots, River Dataset. ${ }^{19}$ This dataset is about the flow measurements of two icelandic rivers collected daily from 1972 to 1974 with temperature and precipitation. Here only the data of 1972 are shown. (a) The diagonal plots show the trends of dimensions over time. (b) The dataset is sorted according to the flow of the Jokulsa Eystri River. The diagonal plots show the possible relations of the flow of the Jokulsa Eystri River with the flow of the Vatnsdalsa River, temperature and precipitation.

1D plots are widely used to show mathematical functions, basic principles in physics, and economic data over time. A 1D Plot can only show one dimensional data. For multivariate data, multiple 1D plots are a very effective tool to reveal trends and relationships. With them, users can read the data in multiple dimensions, can compare the data in multiple dimensions, and can infer the causes for change in one dimension from other dimensions.

Another powerful function of the multiple 1D plots is rearrangement of data points. ${ }^{20}$ The data points may be sorted by the quantitative or categorical order in one dimension, allowing users to search for trends and patterns in the other dimensions. An example is shown in figure 2b. Sorting sometimes partitions the data points into several categories. The category boundary may be vague for quantitative and continuous data dimensions, while it is usually clear for categorical data dimensions. This partitioning makes it possible to compare values, examine trends and find patterns in or between categories.

\subsection{Raster Plots}

Some multivariate data are collected in a regular spatial grid, such as multiple channels of remote sensing data. Raster plots are generally used for the analysis and visualization of this kind of data. A raster plot corresponds to the data of one dimension and it is composed of data points. The data points are put in the plot one by one, from left to right and from top to bottom. If the metadata explicitly specifies the number of the data points in a row (column count), it is used. Otherwise the implicit square root of the record count is used as the raster width. Figure 3 a shows a raster plot whose data are collected from a $128^{*} 128$ grid; the diagonal plots create images of individual dimensions.

The dimension value is mapped to color. There are many possible mapping methods: hue mapping, saturation mapping, lightness mapping, grey scale mapping and customized color mappings in which users specify a color 


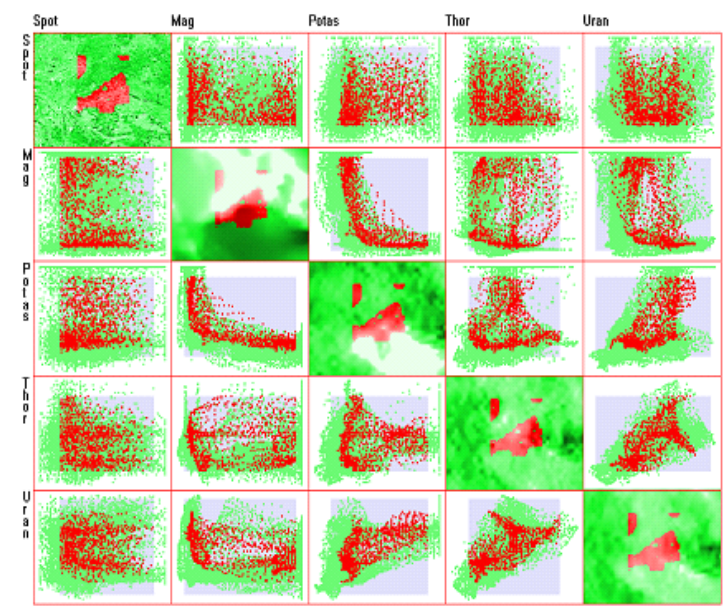

(a)

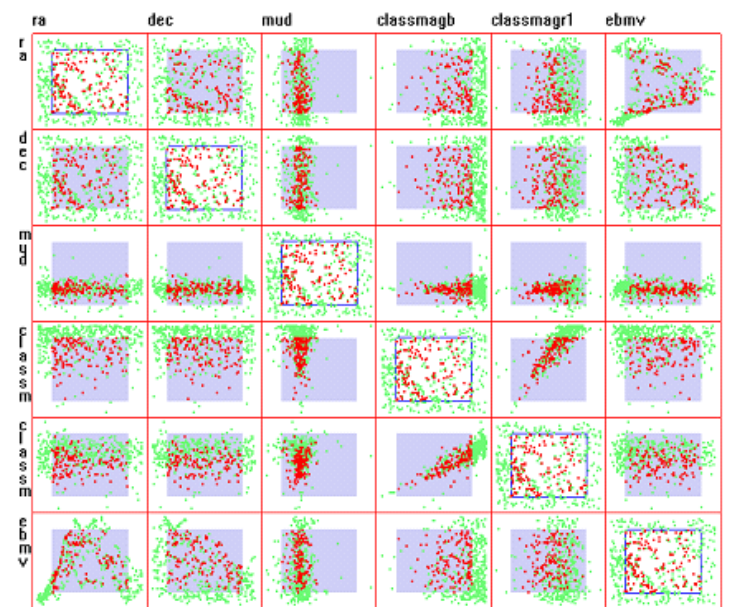

(b)

Figure 3. (a) Scatterplot Matrix with Raster Plots, Out5D Dataset. The diagonal plots show the dataset with raster plots in a $128^{*} 128$ grid. An introduction to out5d dataset can be found in section 4.2. (b) Scatterplot matrix with Data Driven Plots, Astronomy Dataset. The diagonal plots are 2D plots, where the points have explicit location specified by the first two dimensions.

range. The hue mapping, saturation mapping and lightness mapping are based on the HSL (Hue, Saturation and Lightness) color space. For each mapping of these three, one color is selected as the base color. The default base color that we use is fully saturated red for brushed data points, and half saturated green for the regular data points. The different saturation help users with color perception difficulties. The dimension value is normalized into hue, saturation or lightness, and it replaces the corresponding one in the base color to get a new color. The hue mapping, saturation mapping and lightness mapping can be combined together, such as the saturation-lightness mapping.

The grey scale mapping is based on RGB color space. The dimension value is normalized into red, blue and green at equal levels to get the grey color. Customized color mapping allows users to specify a range of color, and the dimension value is normalized and used as an index value into the color map. Some examples of color mappings are shown in figure 4a.

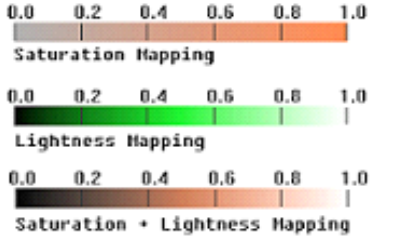

(a)

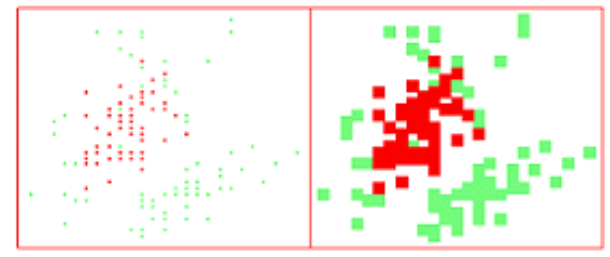

Rectangular, Size=2 Rectangulad, Size=8

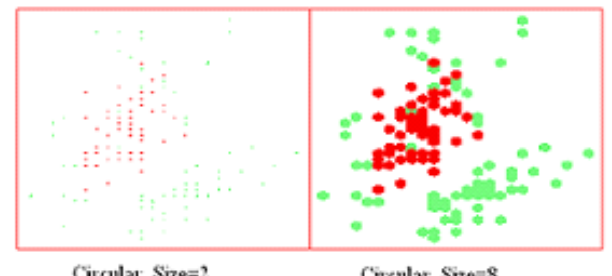

(b)

Figure 4. (a) Color Mappings (b) Point Shape and Size 
The point size can be changed to make the color easy to recognize, fill in the gaps between data points and allow users to reduce overlaps in regular scatterplots. The point shape can be circular or rectangular. Examples are shown in figure $4 \mathrm{~b}$.

\subsection{Data Driven Plots}

Sometimes, the dataset itself contain location attributes, for instance, city climate records may contain the longitude and latitude. Data driven plots are drawn in the diagonal plots for this kind of data. A data driven plot is composed of data points, each of which represents spatial attributes and a dimension value. The spatial attributes is a pair of dimensions, which can be changed through a control panel. Figure 3b shows a data driven plot, in which the dimension ra and dec are used as $\mathrm{x}$ and y coordinate respectively.

In the data driven plots, the dimension value is mapped onto color, employing the same mechanism as that in raster plots. It is basically a hybrid plot, combining the features of raster plots and scatterplots.

\section{BRUSHING AND LINKING}

Brushing is a mechanism by which a user can interactively select a data subset. The selected data may be highlighted, masked, deleted or used for further analysis. Average values of the selected data can be shown, and details about the selected data can be displayed in another window. ${ }^{8}$

The traditional brush operates on data values, in which the brushing condition is based on the dimension value. We extend the brushing operation onto the ordering and spatial attributes of data. Each data record has an associated ordering attribute or spatial attributes. Although the ordering and spatial attributes can be easily described as data values, they are different from data values. They are often implied in the data values, though users can explicitly specify them.

A dataset usually has many potential ordering attributes, such as the data collection order, the temporal order, and the order from sorting the dataset by an individual dimension. When the dataset is retrieved from the database and ordered by distance to the query center, the data sequence of this query will form another order, query-based order. Users can specify one of the orders and explore the dataset by this order.

Usually a dataset only has one set of spatial attributes. The spatial attributes can be explicitly specified by two dimension values, or implicitly specified by the shape of the dataset and the sequence number of the record.

The selection criterion is called the brush constraint, which is used to decide if a record is selected or not. In XmdvTool, the original brush constraint is composed of several atomic conditions for the multivariate dataset. Each atomic condition acts upon a dimension. An atomic condition is a predicate, which has a high value and a low value as its boundaries. It can be described by the following formula:

$$
C_{i}: L_{i} \leq V_{i} \leq H_{i}
$$

Where $C_{i}$ is the atomic condition for the $\mathrm{i}$-th dimension; $L_{i}$ is the lower boundary of the i-th dimension; $H_{i}$ is the upper boundary of the i-th dimension; $V_{i}$ is a data value for the i-th dimension.

Then the brushing constraint for the regular $\mathrm{N}$ dimensional brush can be described by the following formula:

$$
C=\bigcap_{i=1}^{N} C_{i}
$$

where $\mathrm{C}$ is the brushing constraint; $\mathrm{N}$ is the number of dimensions; $C_{i}$ is the $\mathrm{i}$-th atomic condition. The brushing constraint is the product of the all the atomic conditions. This means that the brush will select the data records whose values satisfy all the $\mathrm{N}$ atomic conditions.

\subsection{Brushing on Histograms}

The $\mathrm{X}$-axis of the histogram is the regular data dimension, and the brushing operations on it are like the brushing operations on the scatterplots except they are constrained to the bin boundaries. Users can resize and move the brushing range. The Y-axis of histogram is the number of data points that fall within each bin; there are currently no brushing operations in that direction. The brushing constraint is the same as that in formula 3. 


\subsection{Brushing on the $1 \mathrm{D}$ plot}

The 1D plot employs the ordering attribute and the data dimension as its two dimensions. A new brushing mechanism is introduced, the order-based brush, in which users can operate directly on the ordering attribute to select a range, just as in the brushing operation on the regular data dimensions. The multiple 1D plots share the same brushing range. The selected data points are the data points that fall in the range of the brush. The scatterplots and the 1D plots are linked together, and the changes to the brush in any of them will affect the selection in the others. This brush constraint can be described by the following formula:

$$
C_{1 D}=C_{o} \wedge\left(\bigcap_{i=1}^{N} C_{i}\right)
$$

where $C_{1 D}$ is the brushing constraint; $C_{o}$ is the ordering condition; $\mathrm{N}$ is the number of dimensions; and $C_{i}$ is the i-th atomic condition. The brush constraint is the product of the ordering condition and all the other atomic conditions. It means that the brush will select all the data records in the ordered list whose position satisfy the ordering condition and whose data satisfy all the $\mathrm{N}$ conditions.

\subsection{Brushing on the raster plot}

The data points of a raster plot are structured in a two dimensional space. Users operate on this two dimensional space and get a subspace of two dimensions. Users can resize and move this subspace. The brushed data points are the data points whose spatial attributes fall in this subspace and also satisfy the conditions of the regular brushes. A new brushing mechanism is introduced, the spatial brush, which makes it possible to analyze the spatial attributes of the data. This brush constraint can be described by the following formula:

$$
C_{2 D}=C_{x} \wedge C_{y} \wedge\left(\bigcap_{i=1}^{N} C_{i}\right)
$$

where $C_{2 D}$ is the brushing constraint; $C_{x}$ and $C_{y}$ are the spatial conditions; $\mathrm{N}$ is the number of dimensions; and $C_{i}$ is the i-th atomic condition. The brush constraint is the product of the spatial conditions and all the atomic conditions.

\subsection{Brushing for the data driven plot}

The location attributes of the data records in this plot are provided by two dimensions in the dataset. These two dimensions are like the regular dimensions, and they can be used in the scatterplot matrix. These two dimensions also form a structure space on which the brush can operate to specify a subspace. Like in the raster plot, the brushed data points are the data points that fall in this subspace and also satisfy the conditions of the data brush. This brush has the same constraint as that in formula 5 .

\section{CASE STUDIES}

\subsection{Case Study 1: Time Series Data Analysis}

Many scientists explore time series data in their research. Here we use a wireless network performance dataset ${ }^{21}$ as an example. It is from the Wireless Multimedia Streaming Laboratory in the Computer Science Department at WPI, and describes the measurements of the physical, network and application layers of a streaming video over a wireless campus network. It has many dimensions, and we use six of them: signal_strength (Wireless Signal Strength, physical layer), rtt (Round Trip Time, network layer), lost_rate (Frame Lost Rate, network layer), bandwidth (Bandwidth, network layer), throughput (Throughput, application layer) and framerate (Frame rate, application layer). Four usage patterns illustrate how to explore the time series data with this newly developed tool. 


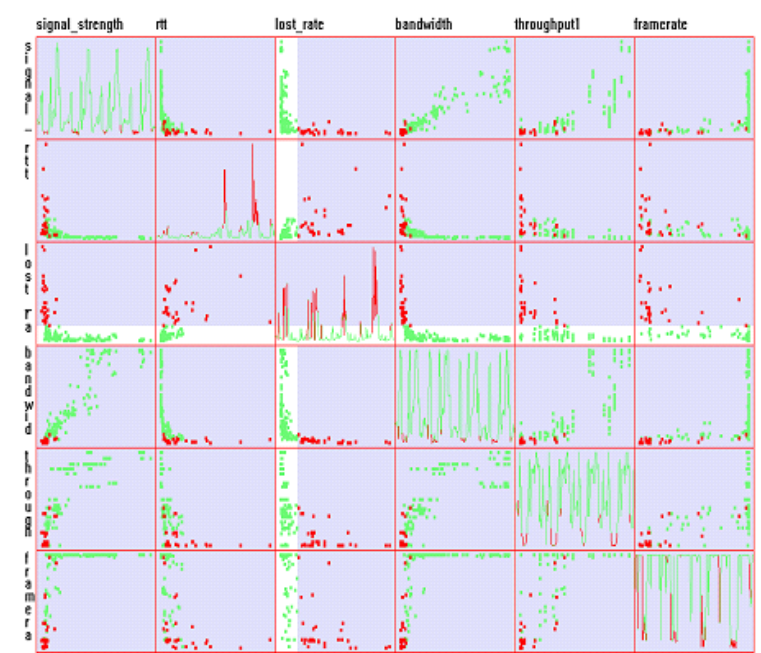

(a)

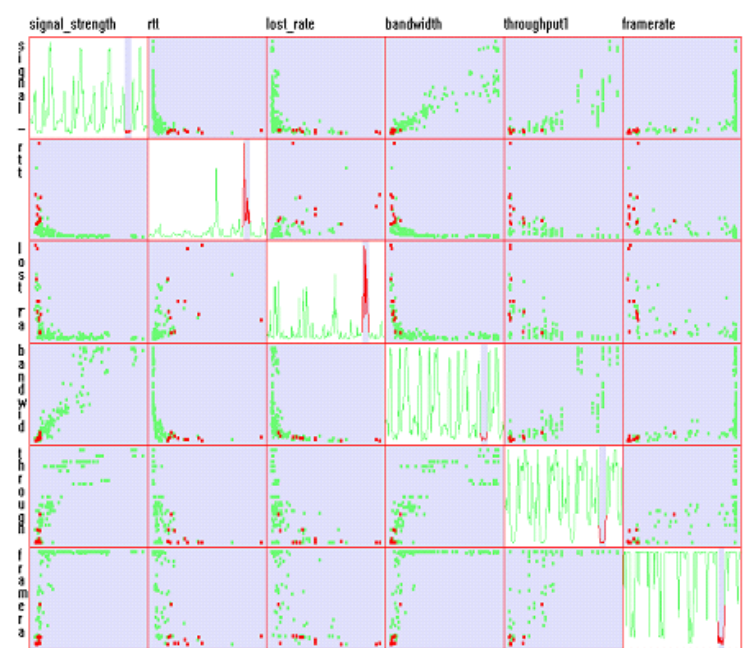

(b)

Figure 5. Time Series Data Analysis. (a) Investigating the dataset from feature space to temporal space. (b) Investigating the dataset from temporal space to feature space.

- Investigating the dataset from feature space to temporal space. We brush a subset of data that has some potentially interesting characteristics in the feature space, and then investigate the distribution in the temporal space. High values of the lost_rate are brushed, and the whole ranges are brushed for other dimensions. The result is shown in figure $5 \mathrm{a}$, with the brushed data marked in red. We can find that four time slots are related to this high lost rate, and they are distributed uniformly in the temporal space.

- Investigating the dataset from temporal space to feature space. We brush a time slot with high values for lost rate, it is shown in figure 5b. Then we observe the characteristics of this dataset in the feature space. In this brushed data subset, framerate value, bandwidth, signal_strength and throughput are low, while rtt and lost_rate are high.

- Observing the trends of every dimension, and comparing the trends among them over the temporal order. From figure 5, we can see that the 1D plots of framerate value, bandwidth, signal strength, and throughput are quite similar; the $1 \mathrm{D}$ plots of lost rate and rtt are somewhat similar. Also with the help of the scatterplots, we conclude that framerate, bandwidth, and throughput have positive correlation with signal strength, and lost rate has positive correlation with rtt.

- Observing the trends of every dimension after sorting the dataset by one dimension. Firstly the dataset is sorted by the signal_strength, and the result is shown in figure 6. From this figure, some trends can be seen very clearly: rtt and lost_rate decrease steadily, and bandwidth, throughput1 and framerate increase steadily along with the increasing of the signal_strength, although there is fluctuation for all of them. Fluctuation may be caused by noise, or it may mean that more factors need to be investigated.

\subsection{Case Study 2: Spatial Data Analysis}

The out5d dataset consists of remote sensing channels, with five attributes: SPOT, magnetics and three radiometrics channels - potassium, thorium and uranium. It was collected from a $128 * 128$ grid of Western Australia. When a dataset has spatial attributes, usually we want to perform the following tasks: finding the spatial distribution of a data subset that has specific characteristics; investigating the characteristics for the dataset in a specific region.

- We show how to carry out the first task. For the magnetics dimension, the upper half range of the values is selected, and for other dimensions, the full ranges are used. This brush is shown in figure 7a, which 


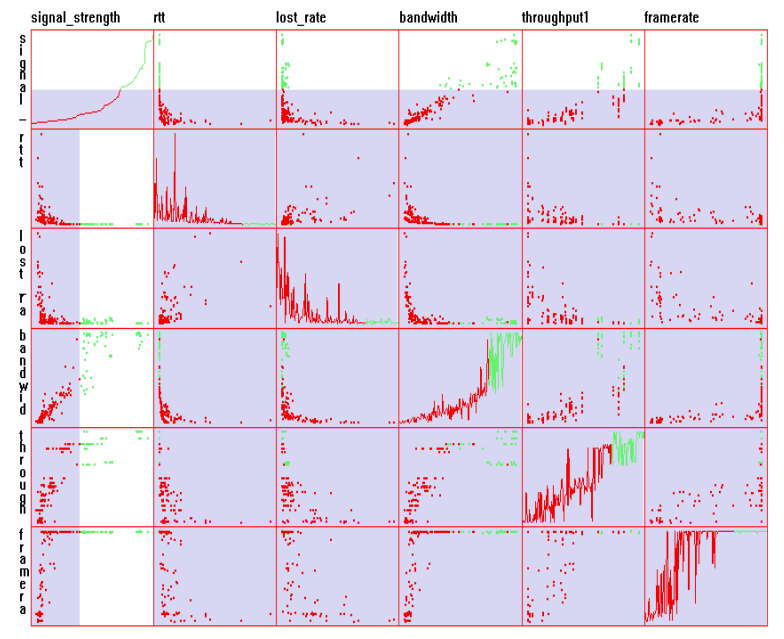

Figure 6. Time Series Data Analysis. Observing the trends of every dimension after sorting the dataset by signal strength.

illustrates the distribution of strong magnetics in geographic space. We can continue to adjust the lower limit of the brush to shorten the brush range, and the result is shown in figure $7 \mathrm{~b}$, which illustrates the distribution of stronger magnetics in geographic space than that in figure 7 a.

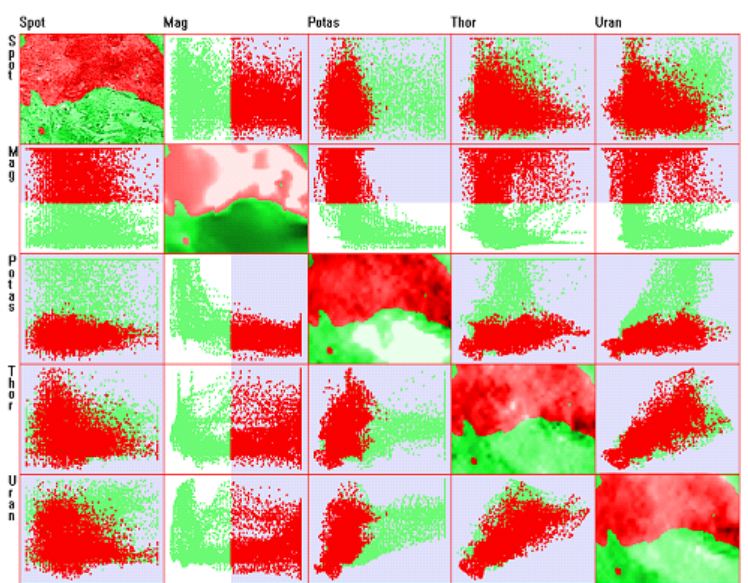

(a)

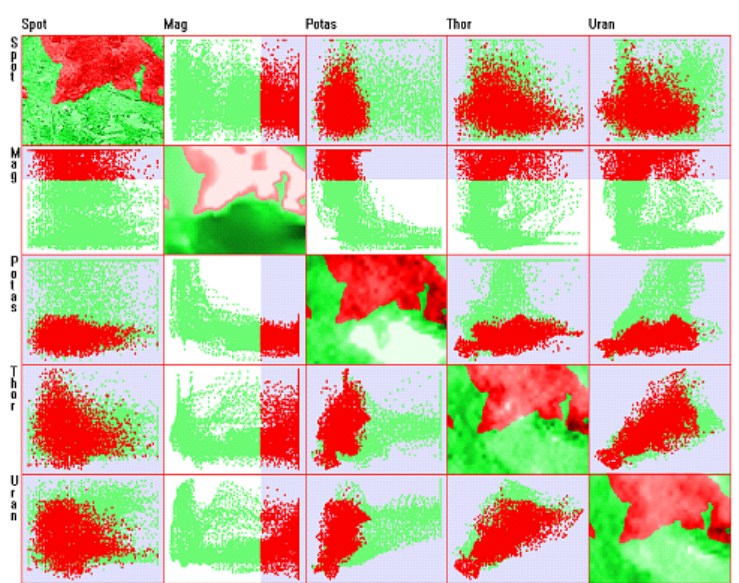

(b)

Figure 7. Spatial Data Analysis. (a) Finding the spatial distribution of data with strong magnetics. (b) Finding the spatial distribution of data with stronger magnetics than that in figure a.

- We show how to perform the second task. The data value is mapped to color. The lighter color marks the higher value. In the Magnetics diagonal plot, there is a very dark zone, which shows that magnetism is low. After brushing this zone with the spatial brush, as shown in figure 8, we can see if this data subset has discernable characteristics. From the scatterplots associated with potassium, we can see that most of the data have high values in potassium, while we cannot find obvious characteristics for the other three dimensions. Thus we can conclude that low values of magnetics has an inverse correlation with potassium in this region, but has no apparent relation with other dimensions in this dataset. 


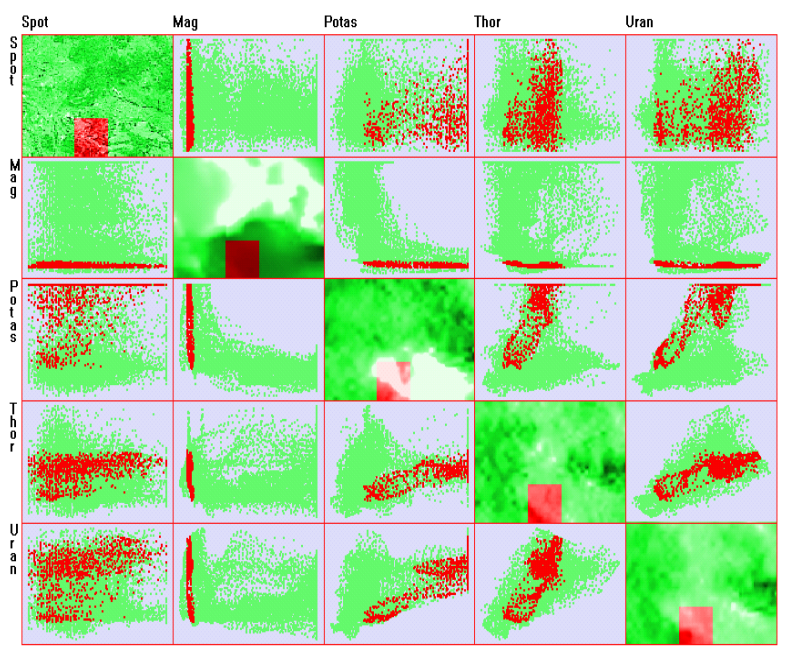

Figure 8. Spatial Data Analysis. Investigating the characteristics for the dataset in a user specified geographic region.

\section{CONCLUSION AND FUTURE WORK}

In this paper, histograms, 1D plots and 2D plots (raster plots and data driven plots) are displayed in the diagonal plots of the scatterplot matrix. The histogram shows the data distribution of one dimension. The 1D plots illustrate the trends of the data along some order, which can be temporal order, spatial order or the order by one dimension. The 2D plots reveal the distribution of the data in two dimensional space.

Furthermore, histograms, 1D plots, 2D plots and scatterplots are linked through brushing. Brushing on one view will affect the other views automatically. Other multivariate data visualization techniques are also linked with these plots, and supplement as effective tools to view the datasets from other perspectives. Two case studies are presented to show how to analyze ordering and spatial datasets with linking and brushing. The integration of histograms, 1D plots and 2D plots with the scatterplot matrix can greatly help to understand datasets and find patterns between feature space and ordering or geographic space.

One future work includes integrating 3D visualization with the scatterplot matrix. 3D plots can show the physical distribution of data points, but it is hard to see all the data points in the screen display. They can become more useful if we integrate them with some interactive methods, such as rotation, zooming, panning, brushing and cut planes. Brushing can also link the 3D plots with the other scatterplots and help users find and understand the data relationship between dimensions and attributes.

Other future work will extend the histograms, 1D plots and 2D plots to the hierarchical scatterplot matrix. XmdvTool has supported hierarchical exploration of large datasets for some time. ${ }^{9}$ We can examine the dataset in multiple levels of abstraction by clustering the data and showing aggregation information for each cluster. XmdvTool also supports hierarchical navigation and filtering. Histograms are easy to integrate with the hierarchical scatterplot matrix, because this integration does not need hierarchical operations. For the 1D plots and 2D plots, we must provide a mechanism for clustering the ordering and spatial attributes and the regular data dimensions at the same time. Then we can show the clusters of the ordering attribute, spatial attributes and data dimension at the same time, and navigate and filter within this hierarchy.

\section{ACKNOWLEDGMENTS}

We appreciate Peter Ketelaar of Geoinformatics Exploration for providing the out5d remote sensing dataset, Prof. Mark Claypool at WPI for providing the wireless network performance dataset, and Dr. Bob Mann at University of Edinburgh for providing the astronomy dataset. 


\section{REFERENCES}

1. W. Cleveland, The Elements of Graphing Data, Wadsworth Inc., 1985.

2. "Nist/sematech e-handbook of statistical methods." http://www.itl.nist.gov/div898/handbook/eda/section3/eda33qb.htm, 2005.

3. M. Ward, "Xmdvtool: Integrating multiple methods for visualizing multivariate data," Proc. IEEE Visualization, pp. 326-333, 1994.

4. A. Inselberg, "The plane with parallel coordinates," Special Issue on Computational Geometry, The Visual Computer 1, pp. 69-97, 1985.

5. D. Andrews, "Plots of high dimensional data," Biometrics 28, pp. 125-136, 1972.

6. J. LeBlanc, M. Ward, and N. Wittels, "Exploring n-dimensional databases," Proc. IEEE Visualization, pp. 230-237, 1990.

7. J. Yang, A. Patro, S. Huang, N. Mehta, M. Ward, and E. Rundensteiner, "Value and relation display for interactive exploration of high dimensional datasets," Proc. IEEE Symposium on Information Visualization , pp. 73-80, 2004.

8. A. Martin and M. Ward, "High dimensional brushing for interactive exploration of multivariate data," Proc. IEEE Visualization, pp. 271-278, 1995.

9. Y. Fua, M. Ward, and E. Rundensteiner, "Navigating hierarchies with structure-based brushes.," Proc. IEEE Symposium on Information Visualization, pp. 58-64, 1999.

10. A. Becker and S. Cleveland, "Brushing scatterplots," Technometrics 29(2), pp. 127-142, 1987.

11. S. Card, J. Mackinlay, and B. Shneiderman, Readings in Information Visualization, Morgan Kaufmann, San Francisco, Calif., 1999.

12. M. K. Tory and T. Moller, "Rethinking visualization: A high-level taxonomy," Proc. IEEE Symposium on Information Visualization, pp. 151-158, 2004.

13. R. Kosara, G. N. Sahling, and H. Hauser, "Linking scientific and information visualization with interactive 3d scatterplots," Proc. International Conference in Central Europe on Computer Graphics, Visualization, and Computer Vision (WSCG), pp. 133-140, 2004.

14. D. L. Gresh, B. E. Rogowitz, R. L. Winslow, D. F. Scollan, and C. K. Yung, "Weave: A system for visually linking 3-d and statistical visualizations, applied to cardiac simulation and measurement data," Proc. IEEE Visualization, pp. 489-492, 2000.

15. A. Goel, C. Baker, C. A. Shaffer, B. Grossman, R. T. Haftka, W. H. Mason, and L. T. Watson, "Vizcraft: A multidimensional visualization tool for aircraft configuration design," Proc. IEEE Visualization, pp. 425428, 1999.

16. G. G. Robertson, J. D. Mackinlay, and S. K. Card, "Cone trees: animated 3d visualizations of hierarchical information," Proc. ACM SIGCHI Conference on Human Factors in Computing Systems, pp. 189-194, 1991.

17. G. Furnas and J. Zacks, "Multitrees: Enriching and reusing hierarchical structure," Proc. ACM SIGCHI Conference on Human Factors in Computing Systems, pp. 330-336, 1994.

18. D. Scott, "On optimal and data-based histograms," Biometrika 66, pp. 605-610, 1979.

19. H. Tong, B. Thanoon, and G. Gudmundsson, "Threshold time series modelling of two icelandic riverflow systems," Water Resources Bulletin 21(4), pp. 651-661, 1985.

20. R. Rao and S. Card, "The table lens: merging graphical and symbolic representations in an interactive focus+context visualization for tabular information," Proc. ACM SIGCHI Conference on Human Factors in Computing Systems, pp. 318-322, 1994.

21. F. Li, J. Chung, M. Li, H. Wu, M. Claypool, and R. Kinicki, "Application, network and link layer measurements of streaming video over a wireless campus network," Proc. Passive and Active Network Measurement Workshop (PAM), pp. 189-202, 2005. 